Abstract 257 Table 1 The odds ratio of having a specific residential expousre among SLE patients and healthy controls, adjusted for race/ethnicity, gender, age and education

\begin{tabular}{|c|c|c|c|}
\hline & $\begin{array}{l}\text { SLE patients } \\
(\mathrm{n}=359)\end{array}$ & $\begin{array}{l}\text { Healthy controls } \\
(\mathrm{n}=106)\end{array}$ & \\
\hline Residential Exposure, ever & $n(\%)$ & $n(\%)$ & $\begin{array}{l}\text { adjusted OR } \\
(95 \% \mathrm{Cl})\end{array}$ \\
\hline Any exposure & $200(56.7)$ & $42(40.0)^{*}$ & $1.9(1.2,3.0)^{*}$ \\
\hline Exterminator ( $>5$ times) & $80(22.3)$ & $12(11.3)^{*}$ & $1.9(1.0,3.7)$ \\
\hline $\begin{array}{l}\text { Home insecticide use } \\
\text { (at least weekly for } 2 \text { months) }\end{array}$ & $73(21.0)$ & $13(12.5)$ & $2.1(1.1,4.1)^{*}$ \\
\hline Kerosene (heater) & $31(8.8)$ & $3(2.9)^{*}$ & $3.0(0.9,10.2)$ \\
\hline Lived in an agricultural area & $103(28.9)$ & $25(24.0)$ & $1.3(0.7,2.1)$ \\
\hline
\end{tabular}

for heating, and living in an agricultural area), and for SLE, disease damage (Brief Index of Lupus Damage, BILD) score and age of lupus diagnosis. Multivariable logistic regression models assessed the association of each exposure with SLE status, and linear regression models to examine the association of each exposure with disease damage (i.e. BILD score) for SLE patients, both adjusting for age, sex, race/ethnicity and education.

Results We included 359 SLE patients with an average diagnosis age of $32 \pm 11$ years and 106 healthy controls. Ninety-one percent of SLE patients and $89 \%$ of controls were female. SLE patients were older than controls, with a mean age of $49 \pm 14$ vs. $41 \pm 16 \quad(\mathrm{p}<0.05)$. Both groups were racially/ethnically diverse, with over $65 \%$ identified as non-White. Over half of SLE patients $(56.7 \%)$ reported any exposure compared to $40 \%$ of controls $(\mathrm{p}<0.05)$. Specific exposures of the SLE group ranged from $8.8 \%$ for kerosene to $28.9 \%$ for agricultural exposure (see table 1). There was a higher prevalence of any exposures among SLE patients vs. controls (OR 1.9, 95\% CI 1.2$3.0, \mathrm{p}<0.05$ ), as well as specific exposures to pesticides (exterminator $)(\mathrm{OR}=1.9,95 \% \mathrm{CI} 1.0-3.7)$, other home insecticide use $\quad(\mathrm{OR}=2.1, \quad 95 \% \mathrm{CI} \quad 1.1-4.1, \quad \mathrm{p}<0.05)$ and kerosene $(\mathrm{OR}=2.8$, 95\% CI 0.8-9.7). We found no association between residential exposures and BILD score in the SLE-only analysis.

Conclusions SLE diagnosis was associated with increased odds of residential exposures, but this study is under powered to fully examine individual exposures. Lack of information on timing of exposures is another study limitation. While this study demonstrates the prevalence of exposures, the observed trend warrants further investigation with assessments of exposure timing relative to age and SLE diagnosis.

Funding Source(s): Centers for Disease Control (U01DP005120)

\section{NK GENE SIGNATURE IN SLE}

${ }^{1}$ Nicole L Fewings*, ${ }^{2}$ Sanjay Swaminathan, ${ }^{3}$ David Booth, ${ }^{4}$ Ming Wei Lin. 'Westmead Institute for Medical Research; ${ }^{2}$ Department of Clinical Immunology, Westmead Hospital, Sydney, New South Wales, Australia Department of Medicine, University of Sydney, Sydney, New South Wales, Australia Department of Medicine, Western Sydney University, Sydney, New South Wal; ${ }^{3}$ Centre for Immunology and Allergy Research, Westmead Institute for Medical Research, University of Sydney, Westmead NSW, Australia; ${ }^{4}$ Dept of Clinical Immunology and Immunopathology, Westmead Hospital, Westmead NSW 2145; and Faculty of Medicine, Sydney Medical School, University of Sydney, NSW 2000

10.1136/lupus-2019-|sm.258
Background SLE has traditionally been considered a disease of dysregulated B cells and the production of pathogenic autoantibodies. Evidence that other cell types contribute to the observed pathology is being recognised with the advent of a new techniques providing focused avenues of exploration. The aim of this study was to determine which genes are differentially expressed in patients compared to healthy an autoimmune controls. This may then be lead to downstream intracellular signalling pathways and aberrant cytokine production which may contribute to the understanding of the pathogenesis of SLE.

Methods Whole blood RNA was extracted from 46 SLE patients with heterogeneous disease manifestations and at different stages of disease activity, 5 Autoimmune encephalitis and 20 healthy controls. Gene expression was measured using a Nanostring nCounter mRNA expression assay incorporating over 500 immunological genes. Data was analysed using Partek Genomics Suite to identify differentially expressed genes and find pathways that may be of interest to interrogate further in the context of SLE.

Results Gene expression analysis unsurprisingly showed higher expression of interferon-related genes in SLE (MX1, GBP1, IRF7, IFIT2), however we also found that there were genes that were under-expressed. An NK signature with genes such as KLRC2, KLRC1, KLRB1, KLRF1, KLRG1, PRF1 and IL2RB shows the potential value of the approach to discern the important cell types in the development of SLE.

Conclusions The most significantly differentially expressed genes in SLE show an NK signature, with genes that encode activating and inhibitory NK receptors having reduced expression compared to healthy controls. This NK signature recognises a role for this arm of the innate immune response as a unique fingerprint of SLE.

Funding Source(s): None

\section{HEALTHCARE SYSTEM AFFECTING SYSTEMIC LUPUS ERYTHEMATOSUS IN ASIA-PACIFIC COUNTRIES}

${ }^{1}$ Chan-Bum Choi* ${ }^{2}$ Shereen Oon, ${ }^{2}$ Mandana Nikpour, ${ }^{3}$ Sang-Cheol Bae. ${ }^{1}$ Hanyang University Hospital for Rheumatic Diseases; ${ }^{2}$ Melbourne University; ${ }^{3}$ Department of Rheumatology, Hanyang University Hospital for Rheumatic Diseases

\subsection{6/lupus-2019-Ism.259}

Background There are between-country disparities in healthcare systems in Asia and Pacific region. The aim of the study was to construct profiles of disparities in healthcare system affecting systemic lupus erythematosus (SLE) in Asia and Pacific countries.

Methods An online survey was conducted between March and October 2018 of rheumatologists specializing in SLE in the Asia and Pacific region. Responses were collected anonymously and analyzed, using descriptive statistics

Results The survey was sent to 45 SLE rheumatologists and $20(44.4 \%)$ provided a complete response. Responders were from 14 countries in the Asia-Pacific region. (Australia, Bangladesh, China, Hong Kong, Indonesia, Japan, Republic of Korea, Kuwait, Myanmar, New Zealand, Philippines, Singapore, Taiwan, Thailand). The estimated prevalence of SLE was 51.1 per 100000 (IQR 26.5 76.9) with 50\% (IQR 36.7 60.0) having lupus nephritis. Most respondents (66.7\%) reported that $80 \%$ to $100 \%$ of the general population had public health insurance. Fifty percent felt that their countrys health care system functions quite well on the whole, and 
there are only a few changes necessary to make it function even better. And another 50\% felt that it functions quite well on the whole, and there are only a few changes necessary to make it function even better. Assessment using a validated disease activity measure for SLE was regularly performed by $66.7 \%$ of the respondents and they all used Systemic Lupus Erythematosus Disease Activity Index (SLEDAI) with 2 responders using both SLEDAI and British Isles Lupus Activity Group (BILAG). Eighty-eight percent responded that mycophenolate mofetil (MMF) was approved for treatment of SLE in their country with $72.2 \%$ responding that it was reimbursed. It was $83.3 \%$ and $94.4 \%$ for intravenous (IV) cyclophosphamide, $50.0 \%$ and $80.0 \%$ for tacrolimus, $72.2 \%$ and $5.6 \%$ for belimumab and $33.3 \%$ and $33.3 \%$ for rituximab, respectively. MMF was most commonly used in induction therapy for lupus nephritis (40.0\%, IQR 25.065 .0$)$, followed by IV cyclophosphamide National Institute of Health (NIH) protocol (20.0\%, IQR 4.0 40.0), IV cyclophosphamide Euro-Lupus Nephritis Trial protocol (15.0\%, IQR 5.0 27.5), tacrolimus plus MMF (2.5\%, IQR 0.0 8.8), tacrolimus (0.0\%, IQR 0.0 $5.0)$, and oral cyclophosphamide (0.0\%, IQR 0.02 .0$)$.

Conclusions There are disparities influencing the management of SLE in Asia and Pacific countries. Some of the recommended treatments for SLE are not approved and not reimbursed for management of SLE in some Asia and Pacific countries.

Funding Source(s): None

\section{MIR-326 PROMOTES RENAL INJURY IN MURINE LUPUS NEPHRITIS}

${ }^{1}$ Yuan Xia*, 'Xuan Fang, ${ }^{1}$ Nan Xiang, 'Xiaojuan Dai, 'Li Jin, 'Xiaomei Li, ${ }^{1}$ Jinhui Tao, ${ }^{2}$ Xiangpei Li. ${ }^{1}$ Department of Rheumatology and Immunology, The First Affiliated Hospital of USTC, Division of Life Sciences and Medicine, University of Science and Technology of China, Hefei, Anhui; ${ }^{2}$ Department of Rheumatology and Immunology, The First Affiliated Hospital of USTC, Division of Life Sciences and Medicine, University of Science and Technology of China, Hefei

\subsection{6/lupus-2019-Ism.260}

Background MicroRNAs play vital role in the immunopathogenesis of human and experimental lupus nephritis, but the contributions of miR-326 to renal injury in systemic lupus erythematosus (SLE) remain to be demonstrated. Here we characterize the function of the miR-326 in MRL/lpr mice of lupus nephritis.

Methods We generated MRL/lpr mice overexpression or silence in miR-326 and analyzed the clinical course of the nephritis with respect to albuminuria. In addition, renal Th17/ Treg cells and IL-17A/TGF- expression were detected by flow cytometry and immune-histochemistry respectively.

Results miR-326 overexpression did increase the development of albuminuria in MRL/lpr mice. In contrast, miR-326 silence decreased the development of albuminuria. The characterization of renal CD4 $+\mathrm{T}$ cells in miR-326 overexpression mice revealed high numbers of infiltrating Th17 cells and low numbers of infiltrating Tregs. IL-17A and TGF- expression respectively increased and decreased in miR-326 overexpression mice.

Conclusions miR-326 overexpression plays major role in the immunopathogenesis of lupus nephritis in MRL/lpr mice. Thus, our results suggest that miR-326 may be an intriguing new therapeutic approach for patients with lupus nephritis.
Funding Source(s): the National Natural Science Foundation of China 81373186

\section{MIR-326 REGULATES CD4+T CELLS DIFFERENTIATION IN LUPUS DISEASE OF MRL/LPR MICE}

${ }^{1}$ Yuan Xia*, 'Xuan Fang, ${ }^{1}$ Nan Xiang, ${ }^{1}$ Xiaojuan Dai, ${ }^{1}$ Li Jin, ${ }^{1}$ Xiaomei Li, ${ }^{2}$ Jinhui Tao, ${ }^{3}$ Xiangpei Li. ${ }^{\prime}$ Department of Rheumatology and Immunology, The First Affiliated Hospital of USTC, Division of Life Sciences and Medicine, University of Science and Technology of China; ${ }^{2}$ Department of Rheumatology and Immunology, The First Affiliated Hospital of USTC, Division of Life Sciences and Medicine, University of Science and Technology of China, Hefei, Anhui; ${ }^{3}$ Department of Rheumatology and Immunology, The First Affiliated Hospital of USTC, Division of Life Sciences and Medicine, University of Science and Technology of China, Hefei

\subsection{6/lupus-2019-Ism.261}

Background CD4 + T cells play a major role in systemic lupus erythematous (SLE). Many aberrations in miR-326 expression have been described as related to abnormal $\mathrm{T}$ cell activation in SLE. The aim of this study was to investigate the effect of miR-326 expression on the differentiation of CD4 $+\mathrm{T}$ cells in MRL/lpr mice.

Methods 3 groups of female MRL/lpr mice were injected with lentivirus-miR-326 (LV-326) or lentivirus-miR-326 specific inhibitor (LV-sponge) to increase or inhibit miR-326 expression, respectively, and lentivirus-no-encoding (LV-ctrl) as control10 mice per group. The percentage of Th17, Th1, and Treg cells in spleen were determined by flow cytometry, the expression levels of CD4 +T related cytokines were determined by CBA and ELISA.

Results The results showed that, compared with LV-ctrl mice and LV-sponge mice, LV-326 mice had higher percentage of Th17 cells, and lower percentage of Tregs and Th1 cells in splenic CD4 + T cells. In contrast, LV-sponge mice had lower percentage of Th17 cells as well as higher percentage of Tregs and Th1 cells than LV-ctrl mice in splenic CD4 $+\mathrm{T}$ cells. Moreover, serum levels of IL-17A were significantly increased in LV-326 mice, compared with LV-ctrl mice and LV-sponge mice. Serum levels of IL-2 and TGF- were decreased in LV326 mice compared with LV-ctrl mice.

Conclusions These findings suggesting that miR-326 regulates CD4 + T cells differentiation and inflammatory-related cytokines production in lupus model mouse. Implying that miR326 may play a vital role in SLE pathogenesis by regulating CD4 + T cells differentiation.

Funding Source(s): the National Natural Science Foundation of China (81373186)

\section{IMMUNOLOGICAL PATHWAYS IN SYSTEMIC LUPUS ERYTHEMATOSUS DISEASE MANISFESTAION: CEREBRAL LUPUS}

${ }^{1}$ Nicole L Fewings*, ${ }^{2}$ Sanjay Swaminathan, ${ }^{3}$ David Booth, ${ }^{4}$ Ming Wei Lin. 'Westmead Institute for Medical Research; ${ }^{2}$ Department of Clinical Immunology, Westmead Hospital, Sydney, New South Wales, Australia Department of Medicine, University of Sydney, Sydney, New South Wales, Australia Department of Medicine, Western Sydney University, Sydney, New South Wal; ${ }^{3}$ Centre for Immunology and Allergy Research, Westmead Institute for Medical Research, University of Sydney, Westmead NSW, Australia; ${ }^{4}$ Dept of Clinical Immunology and Immunopathology, Westmead Hospital, Westmead NSW 2145; and Faculty of Medicine, Sydney Medical School, University of Sydney, NSW 2000

10.1136/lupus-2019-Ism.262 